\title{
MS22-03 | Electron Densities Of two NonApetides from InVARIOM Application
}

Luger, Peter (Free University Berlin, Berlin, GER); Dittrich, Birger (Heinrich-Heine-University, Düsseldorf, GER)

The invariom formalism and the related database of aspherical scattering factors were introduced more than a decade before. Like other database approaches the invariom formalism relies on Richard Bader's transferability concept of atomic or submolecular fragments. Since transferability was originally examined for single amino acids and later for oligopeptides, invariom applications on oligo- and polypeptides became of early interest. We report here an invariom study on the rare case of two nonapeptides where very accurate X-ray data sets to $(\sin \theta / \lambda)_{\max }=$ $0.61 \AA^{-1}$ were published [1].The compounds

Cyclo (Val-Leu-Pro-Ile-Leu-Leu-Leu-Val-Leu) (I); CSD code LETPIM;

Cyclo (Val-Leu-Pro-Ala-Leu-Leu-Leu-Val-Leu) (II); CSD code LETHIE

were identified as candidates for development as potential anti-cancer drugs. They differ only in residue 4, Ile in (I) and Ala in (II). The structures are free of disorder, a favorable (but not essential) provision for invariom treatment. It was therefore a task of a few mouseclicks to generate and refine an invariom based electron density and to derive results. Focus was directed to discuss molecular surfaces like electrostatic and Hirshfeld surfaces. Both nonapeptides are composed exclusively of non-polar amino acid residues. Consequently the outer electrostatic potential surfaces show mostly positive regions, negative potential is seen in the interior of the cyclic peptides. Since the anti-tumor potency is reduced for the Ala peptide (II), the question how the exchange at residue 4 influences the electron density, will be examined.

[1] Bioorg. Med. Chem. 26 (2018), 609-622. 